\title{
Steroid induced diabetes mellitus in patients receiving prednisolone for haematological disorders
}

\author{
Kotila $\mathrm{TR}^{1}$, Olutogun $\mathrm{T}^{1}$, ${ }^{*}$ Ipadeola $\mathrm{A}^{2}$
}

1. Department of Haematology, College of Medicine, University of Ibadan, Ibadan, Nigeria

2. Department of Medicine, College of Medicine, University of Ibadan, Ibadan, Nigeria

\begin{abstract}
Introduction: Steroids are a useful component of combination chemotherapy or as a single agent in the treatment of haematological disorders even though there are adverse effects associated with its use.

Methods: We report four patients who developed diabetes mellitus (DM) during treatment with steroids for haematological disorders despite a negative history of DM.

Results: The mean age of the patients was 55yrs and DM was diagnosed by fasting plasma glucose (FPG) after a cumulative steroid dose of 500-1800mg.

Conclusion: It is necessary to have a baseline and frequent FPG in patients who are considered for combination chemotherapy which include steroid since the development of DM does not appear to be dose dependent or related to history of DM in the patient or family.
\end{abstract}

Key words: Steroid, Diabetes Mellitus, Haematological malignancy, Chemotherapy, Adverse effect

African Health Sciences 2013; 13(3): 842 - 844 http://dx.doi.org/10.4314/ahs.v13i3.45

\section{Introduction}

Combination chemotherapy is useful in the management of haematological malignancies and this evolved from the use of single agents in order to overcome drug resistance. Attention was drawn to the benefit of steroids in the treatment of lymphoid malignancy by the observation that it induces regression of lymphoid tumours in $\operatorname{man}^{1}$. Subsequently, massive doses of steroids were used in the management of haematological malignancies but the associated side effects preclude its routine $u^{2} e^{2}$. The use of physiological doses with treatable side effects heralded its use in combination chemotherapy ${ }^{3}$.

Steroids continued to be used as an adjuvant in most of these drug combinations and in pulses despite known complications associated with its use. Steroid use is not only limited to haematological disorders but it is also useful as an immunosuppressant in other disease conditions and especially now in transplantation medicine. Adverse effects associated with the use of steroids include gastritis, glaucoma, hypertension and impaired

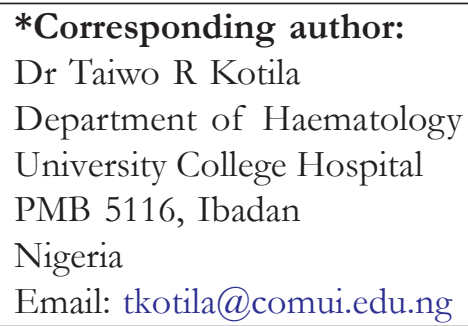

glucose tolerance, there are reports on these adverse effects in its use in the treatment of many disease conditions $^{4-6}$ but we are not aware of any report on the side effects of steroids in combination chemotherapy for haematological disorders.

We discuss four patients who developed Diabetes Mellitus (DM) after the use of combination chemotherapy which included Prednisolone for the treatment of haematological disorders.

\section{Patients}

The age range of the patients was 43-66 years, with a mean of 55 years and included three female and one male. Two of the patients were managed for lymphoma while the other two were managed for chronic lymphocytic leukaemia and aplastic anaemia. The lymphoma patients had combination chemotherapy with Cyclophosphomide, Hydroxydaunorubicin, Oncovin and Prednisolone (CHOP) while the patient with chronic leukaemia had Cyclophosphmide, Oncovin and Prednisolone (CVP). The patient with apalastic anaemia had Prednisolone alone. All the patients on combination chemotherapy received $60 \mathrm{mg}$ of Prednisolone daily for five days with each cycle of the chemotherapy while the patient with aplastic anemia had same dose daily until the diagnosis of DM after which the dose was reduced to $30 \mathrm{mg}$ daily. All the patients denied any history of diabetes in the past in the family. Two of the patients had normal fasting plasma glucose

African Health Sciences Vol 13 Issue 3 September 2013 
(FPG) before commencing chemotherapy but the other two patients did not have the test done. The diagnosis of diabetes mellitus was based on FPG which is $>126 \mathrm{mg} / \mathrm{dl}$ or a random glucose concentration exceeding $200 \mathrm{mg} / \mathrm{dL}$ at least twice after beginning steroid treatment and these patients had FPGs between 147-566mg/dl. Normoglycaemia was achieved with insulin therapy and patients were maintained on oral glucose lowering agents. There was poor response to chemotherapy in all the patients. One of the patients with lymphoma was diagnosed as hypertensive two months before the diagnosis of lymphoma while the patient with aplastic anaemia also developed hypertension while on steroid therapy. Others had no history of hypertension and remained normotensive thereafter.

\section{Discussion}

Diabetes is a known complication of steroid therapy whether as a single agent or in combination with other drugs as seen in our group of patients. The diabetogenic effect of glucocorticoids is said to be determined by dose, duration of administration and type of steroid. Diabetes has also been associated with L- asparaginase in combination chemotherapy when used in the treatment of acute leukaemia and often it is used along with steroid ${ }^{7,8}$ but the diabetes resulting from the use of $\mathrm{L}$-asparaginase is transient.

The transient effect may be because the reported cases are children and also because it is used for a short period as induction chemotherapy, in contrast to our patients who are adults and in whom the use of steroid is for longer periods. This complication was also observed in patients in the middle age with a predilection for female (though only four patients were seen), which is similar to what was observed in a group of patients treated with steroid for varied respiratory diseases ${ }^{4}$. A common finding in cases of steroid induced diabetes mellitus (SIDM) is that it appears not to be dose related and risk factors are not readily identified even though some studies have alluded to a higher prevalence in people of black ancestry but this is yet to be confirmed ${ }^{9,10}$. The cumulative dose until the diagnosis of DM ranged between $500-1800 \mathrm{mg}$ in our group of patients while in the patients who developed SIDM after treatment for respiratory diseases the cumulative dose ranged between 940$23590 \mathrm{mg}$.

The patient with aplastic anaemia had the steroid for less than ten days before DM was confirmed but unfortunately he did not have a baseline test done before the commencement of steroid .He denied previous history of DM but the early onset of DM here may suggest that this patient likely had a significant degree of glucose intolerance prior to administration of steroids. The other patients had the treatment with steroid for between 2-6mths before the onset of DM suggesting that the length of therapy before development of SIDM is highly individualized and is affected by other risk factors. Some reports have suggested that SIDM affects persons in the older age group which a number of the patients in the study fall into. This is due to the decreased ability of the islets cells of Langerhans to control insulin resistance induced by glucocorticoids with increasing age.

The use of steroids in renal transplant patients is also associated with DM as a side effect even when used at low doses but the length of use is longer (in years). The incidence of SIDM reduced from $20 \%$ in a study that gave $5-7.5 \mathrm{mg} /$ day for maintenance over a five year period ${ }^{10}$ to $7 \%$ in another study that prescribed $2.5 \mathrm{mg} /$ day for two years ${ }^{11}$. The non use of steroids as maintenance agents is however being advocated for, in renal transplant patients because of the associated side effects ${ }^{10}$. There is a need to consider the non use of steroid in combination chemotherapy for haematological disorder if SIDM is reported from other centres and after a controlled trial confirms the finding. It is also necessary to identify other risk factors associated with the development of SIDM while the role of age and ethnicity should be further explored.

Physiologic doses are used in pulses in combination chemotherapy for haematological malignancies while low continuous doses are used in renal transplant patients yet these patients still develop SIDM. It is therefore evident that the dose, whether as low continuous or in pulses does not prevent the adverse effect of developing DM.

\section{Conclusion}

If possible, the removal of steroids or the use of other immunosuppressant may be considered not only for haematological disorders but also for chronic disorders that require long term use of steroids. However, when steroids have to be used, there is a need for frequent blood glucose monitoring, so that rising blood glucose values can be detected early and appropriate therapy instituted. We advocate that this 
should be done after each cycle or before the commencement of another cycle.

Early intervention will prevent the onset of acute complications of DM as well as possible long term complications, thus reducing morbidity and mortality associated with SIDM as a complication of therapy. Further prospective studies or randomized controlled trials need to be carried out to accurately outline the long term advantages and disadvantages of steroid therapy in persons requiring treatment with these drugs for survival.

\section{References}

1. Pearson OH, Eliel LP. Adrenocorticotrophic hormone and cortisone induced regression of lymphoid tumors in man: a preliminary report. Cancer 1949;2(6):943945

2. Ranney HM, Gellhorn A. The effect of massive prednisone and prednisolone therapy in acute leukemia and malignant lymphomas. American Journal of Medicine 1957;22:405-413

3. Hall TC, Choi OS, Abadi A, Krant MJ. High dose corticoid therapy in Hodgkin's disease and otherlymphomas. Annals of Internal Medicine 1967;66(6):1144-1153

4. Kim YK, Yoo C, Lee CT, Chung HS, Kim YW, Han SK. Incidence and risk factors of steroid-induced Diabetes in patients with respiratory disease. Journal of Korean Medical Science 2011;26:264-267
5. Papang R, John AS, Abraham S, Rao PS. A study of steroid induced diabetes mellitus in leprosy. Indian Journal of Leprosy 2009; 81(3):12512-12519

6. Tripathi RC, Parapuram SK, Tripathi BJ, Zhong Y, Chalam KV. Corticosteroids and glaucoma risk. Drugs Aging 1999;15(6):439-450

7. Alves C, Chaves C, Souza M. Transient diabetes mellitus related to L-asparaginase therapy. Arquivos Brasileiros de Endocrinologia e Metabologia 2007;51(4):635-638

8. Wang YJ, Chu HY, Shu SG, Chi CS, Hyperglycaemia induced by chemotherapeutic agents used in acute lymphoblastic leukaemia: report of 3 cases. Zhonghua Sheng Wu Yi Xue Gong Cheng Za Zhi (Taipei) 1993;51(6):457-461

9. Odocha O, McCauley V, Scantlebury R, Shapiro P, Caroll M, Jordan C et al. Posttransplant diabetes mellitus in African Americans after renal transplantation under FK506 immunosuppression. Transplantation Proceedings 1993;25(4):2433-2434

10. Gallon LG, Winoto J, Leventhal JR, Parker MA, Kaufman DB. Effect of prednisone versus No prednisone as part of maintenance immunosuppression on long term renal transplant function. Clinical Journal of the American Society of Nephrology 1 2006;1029-1038

11. Kishikawa H, Nishimura K, Soda T, Yamanaka K, Hirai T, Kyo M et al. Low-dose steroid maintenance for renal transplant recipient. Transplantation Proceedings 2010;42(10):4030-4032 